University of Nebraska - Lincoln

DigitalCommons@University of Nebraska - Lincoln

Uniformed Services University of the Health

Sciences

U.S. Department of Defense

2005

\title{
Factor structure and construct validity of the Anxiety Sensitivity Index among island Puerto Ricans
}

\author{
Jennifer A. Cintrón \\ American University \\ Michele M. Carter \\ American University \\ Sonia Suchday \\ Albert Einstein College of Medicine \\ Tracy Sbrocco \\ Uniformed Services University of the Health Sciences \\ James Gray \\ American University
}

Follow this and additional works at: https://digitalcommons.unl.edu/usuhs

Part of the Medicine and Health Sciences Commons

Cintrón, Jennifer A.; Carter, Michele M.; Suchday, Sonia; Sbrocco, Tracy; and Gray, James, "Factor structure and construct validity of the Anxiety Sensitivity Index among island Puerto Ricans" (2005). Uniformed Services University of the Health Sciences. 21.

https://digitalcommons.unl.edu/usuhs/21

This Article is brought to you for free and open access by the U.S. Department of Defense at DigitalCommons@University of Nebraska - Lincoln. It has been accepted for inclusion in Uniformed Services University of the Health Sciences by an authorized administrator of DigitalCommons@University of Nebraska Lincoln. 


\title{
Factor structure and construct validity of the Anxiety Sensitivity Index among island Puerto Ricans
}

\author{
Jennifer A. Cintrón ${ }^{\mathrm{a}, *}$, Michele M. Carter ${ }^{\mathrm{a}}$, \\ Sonia Suchday ${ }^{\mathrm{b}}$, Tracy Sbrocco ${ }^{\mathrm{c}}$, James Gray ${ }^{\mathrm{a}}$ \\ ${ }^{a}$ Department of Psychology, American University, 321 Asbury Bldg., \\ Washington, DC 20016-8062, USA \\ ${ }^{\mathrm{b}}$ Albert Einstein College of Medicine, New York, NY, USA \\ ${ }^{\mathrm{c}}$ Uniformed Services University of the Health Sciences, \\ Bethesda, MD, USA
}

Received 30 June 2003; received in revised form 25 August 2003; accepted 31 October 2003

\begin{abstract}
The factor structure and convergent and discriminant validity of the Anxiety Sensitivity Index (ASI) were examined among a sample of 275 island Puerto Ricans. Results from a confirmatory factor analysis (CFA) comparing our data to factor solutions commonly reported as representative of European American and Spanish populations indicated a poor fit. A subsequent exploratory factor analysis (EFA) indicated that a two-factor solution (Factor 1, Anxiety Sensitivity; Factor 2, Emotional Concerns) provided the best fit. Correlations between the ASI and anxiety measures were moderately high providing evidence of convergent validity, while correlations between the ASI and BDI were significantly lower providing evidence of discriminant validity. Scores on all measures were positively correlated with acculturation, suggesting that those who ascribe to more traditional Hispanic culture report elevated anxiety.
\end{abstract}

(C) 2003 Elsevier Inc. All rights reserved.

Keywords: Anxiety sensitivity; Factor analysis; Hispanics; Puerto Ricans

\footnotetext{
* Corresponding author.

E-mail address: Jennycintron@yahoo.com (J.A. Cintrón). 


\section{Introduction}

Ethnicity has been suggested to be a potentially significant variable in the differential expression of psychopathology (Carter, Miller, Sbrocco, Suchday, \& Lewis, 1999). For example, Carter et al. (1999) found that African Americans differ slightly from European Americans in the features that characterize anxiety sensitivity. Specifically, they noted that although anxiety sensitivity among African Americans is multidimensional, number and composition of the anxiety sensitivity dimensions exhibited by African Americans differ from those characteristic of European Americans.

Similarly, Smith, Friedman, and Nevid's (1999) study on phenomenological differences between African American and European American patients with panic disorder with or without agoraphobia illustrates the plausible presence of ethnic differences in symptomatology. Results suggest that African American patients with panic disorder demonstrated a significantly later age of onset, and showed higher rates of PTSD comorbidity. In addition, African Americans reported greater panic attack symptom severity with more intense levels of numbing or tingling in the extremities, as well as a greater fear of dying or going crazy.

Empirical investigations examining the impact of Hispanic culture on psychopathology and assessment, however, have been limited in scope (Ortega, Rosenheck, Alegria, \& Desai, 2000). In addition, several studies reported in the extant literature have ignored the subtle differences that may exist among Hispanic groups. This study aimed to address some of these gaps in the literature by examining influence of acculturation on the construct of anxiety sensitivity among island Puerto Ricans.

\subsection{Deficit of epidemiologic data among Hispanic populations}

Examining the relationship between ethnicity and the development of psychopathology is increasingly important among Hispanics, as this population is currently the largest minority group in the United States (U.S. Bureau of the Census, 2003). The rapid growth of the Hispanic population has prompted the increased attention given to mental health issues specific to this population. In spite of the amount of evidence suggesting that ethnicity might play an important role in the epidemiology and differential expression of psychopathology (Carter, Sbrocco, \& Carter, 1996; Carter et al., 1999), literature investigating the nature of psychological disorders among Hispanics has been limited in depth and comprehensiveness (Ortega et al., 2000).

To date, the most extensive source of data pertaining to ethnic minorities is the Epidemiologic Catchment Area (ECA) Program. Findings from the Los Angeles Epidemiologic Catchment Area Project (LAECA) compared prevalence of mental disorders between Mexican-Americans and non-Hispanic Whites and revealed that rates for most mental disorders were strikingly equivalent for both 
ethnic groups (Karno, Burnam, Hough, Escobar, \& Golding, 1987). Contrary to the LAECA conclusions, Mirowsky and Ross (1987) reported significant ethnic differences in reported levels of distress. Specifically, it was determined that symptoms typically associated with malaise, anxiety and depression differ between Mexican and Anglo individuals. Overall results of this study suggested that individuals raised in Mexico report lower levels of distress compared to Mexican-Americans raised in the U.S., who in turn report less distress than Anglo individuals. Mirowsky and Ross (1987) posit that each culture's emphasis on personal survival strategies was a key factor in the observed differences. Specifically, while Anglo culture stresses the independence and individuality of its members, Mexican culture emphasizes the importance of loyalty within interpersonal relationships and collectivism.

Dohrenwend (1969) conducted one of the first studies examining the incidence of psychiatric disorders, particularly rates of depressive symptoms, among African American, Jewish, Irish, and Puerto Rican individuals. Mainland Puerto Ricans reported significantly higher rates of depressive symptoms than did their counterparts, even after socioeconomic status was controlled. This conclusion has also been supported by other investigations over the last three decades (Escobar et al., 1983; Gaw, 1993; Haberman, 1976). Canino et al. (1987), however, found that an island-wide survey of mental disorders in Puerto Rico did not support the notion that Hispanics have higher psychopathology rates than non-Hispanics. An exception to this was a higher prevalence rate of somatization disorders among Puerto Ricans in relation to U.S. community members.

This strong somatization pattern was also reported by Sylva (1997) in a series of case studies of Puerto Rican, non-Hispanic Black, and non-Hispanic White women in New York City. Puerto Rican women displayed more severe somatic and psychological complaints than non-Hispanic Black women, who in turn display higher levels of somatic and psychological complaints than non-Hispanic White women. Sylva (1997) proposed that the inter-ethnic differences observed are associated with the socioeconomic and demographic disparity among groups. For instance, less education and a lower household income significantly increased the likelihood of both somatic and psychological complaints. Once these variables were controlled the only observable difference between Puerto Ricans and nonHispanic Whites was a greater incidence of somatic complaints by Puerto Rican women. It has been suggest in previous investigations that somatization may represent a culturally sanctioned method for eliciting social and emotional support (Duran, 1995).

Studies examining the associations between Hispanic ethnicity and the risk of psychiatric disorders have been criticized because of two main limitations. First, social stressors have been overlooked (Ortega et al., 2000). For most Hispanics, the migration experience is synonymous with the estrangement from supportive interpersonal bonds (Rogler, Gurak, \& Santana Cooney, 1987). And, second, earlier studies have not taken into account that there is significant diversity among 
Hispanic groups, including socioeconomic status and other demographic characteristics such as country of origin, urban or rural residence, and "specific cultural elements historically rooted in their respective nationalities" (Aponte \& Wohl, 2000; Rogler, Cortes, \& Malgady, 1991; Straussner, 2001). By subsuming different ethnocultural groups into one, important factors remain unexamined, as it is erroneously assumed that Hispanic groups may be viewed interchangeably and that generalizations can be applied to all (Flaherty, 1987). As such, this investigation was designed to focus solely on the manifestation of anxiety sensitivity among island Puerto Ricans.

\subsection{Anxiety sensitivity}

Anxiety sensitivity (AS), defined as the fear of anxiety-related symptoms that result from the belief that these symptoms have harmful somatic, social, or psychological consequences, is said to be an amplification of the fear responding factor (Taylor, Koch, McNally, \& Crockett, 1992). When individuals high in anxiety sensitivity become anxious, they are more likely to misinterpret the symptoms brought about by the anxiety, believing that these will have detrimental consequences. This produces the development of a cycle, whereby onset of initial anxiety produces additional anxiety, and may eventually result in the experience of a panic attack (Reiss, 1991; Carter et al., 1999).

Additionally, empirical research has shown that elevated AS constitutes as a cognitive risk factor in the development and maintenance of anxiety disorders (Maller \& Reiss, 1992). Notably, it has been found that relative to participants low in AS, those individuals scoring high in AS are more likely to experience a panic attack and develop an anxiety disorder. Thus, as the predictability associated with AS serves as a way to anticipate panic-like reactions, one may speculate the presence of a possible link between ataque de nervios and elevated levels of AS among individuals who report the presence of ataques. Specifically, in the same way that elevated levels of AS have been shown to precede the development of panic attacks (Donnell \& McNally, 1990), elevated AS may similarly precede onset of ataques. It is imperative to first consider the generalizability of the anxiety sensitivity construct across cultural profiles.

Findings from studies examining the factor structure of the Anxiety Sensitivity Index (ASI) among a mostly European American sample revealed a hierarchical factor structure with three discrete first-order factors (Mental Incapacitation Concerns, Physical Concerns, and Social Concerns) and a general higher order factor (Mohlman \& Zinbarg, 2000; Zinbarg and Barlow, 1996; Zinbarg, Mohlman, \& Hong, 1999). Conversely, in attempting to validate the construct of anxiety sensitivity among Spanish clinical populations, Sandin, Chorot, and McNally (1996) found evidence for a unifactorial structure of the Spanish ASI. Sandin and colleagues (2001) further replicated this finding when investigating the joint factor structure of the ASI and the trait version of the State-Trait 
Anxiety Inventory (STAI-T). As expected, a factor analysis yielded two distinct factors, one for each scale, further supporting the notion that anxiety sensitivity differs from trait anxiety and providing evidence that the ASI measures a single construct.

More recently, Zvolensky and colleagues (2003) found that a two-factorial solution for the Anxiety Sensitivity Index-Revised (ASI-R) was the most replicable across six different countries (Canada, France, Mexico, Spain, The Netherlands, and USA). Specifically, in each country, the two-factorial solution provided the best fit yielding a factor reflecting Fear of Somatic Symptoms (including cardiovascular, respiratory, and gastrointestinal) and a second reflecting Social-Cognitive Concerns. Based on study findings, the authors vigilantly suggest the uniformity of AS across geographical, linguistic and cultural backgrounds.

If, as Zvolensky et al. (2003) suggest, AS cuts across geographical, linguistic, and cultural boundaries, then we would expect that Sandin et al.'s $(1996,2001)$ unifactorial ASI structure be replicated among analogous groups. However, caution should be exercised when making such an assumption, as Spaniards may not be representative of all Hispanic groups. Furthermore, although Spanish language serves as a common link between Spaniards and Puerto Ricans, factors such as geographic location, economic circumstances, national history, and political status, should not be underestimated in the degree of influence they have on the development of cultural identities and culturally specific manifestations of anxiety pathology.

In light of the apparent importance of anxiety sensitivity as a predisposing factor for the development of anxious pathology and the paucity of information currently available regarding anxiety sensitivity among Puerto Ricans, this investigation sought to explore the factor structure of the ASI among island Puerto Ricans, particularly in relationship to acculturation. Comparisons of the factor structure for Puerto Ricans was expected to be a better fit with the structure reported for Spaniards than that reported for mostly European American samples. Furthermore, it was predicted that anxiety level would be positively correlated with an acculturation measure. In other words, higher enculturation levels would parallel higher anxiety scores.

\section{Method}

\subsection{Participants}

Two hundred and seventy-five Puerto Ricans currently residing in a metropolitan area of Puerto Rico participated in the current study. Participants ethnically self-identified as Puerto Ricans, and were 18 years of age or older. Participants who self-identified as other than Puerto Rican $(n=39)$ were excluded from the study in order to maintain cultural homogeneity among the sample. Participants born outside Puerto Rico, but who currently resided on the 
Table 1

Acculturation characteristics

\begin{tabular}{|c|c|c|}
\hline Characteristic & $n$ & $\%$ \\
\hline \multicolumn{3}{|l|}{ PAS } \\
\hline \multicolumn{3}{|l|}{ Psychological acculturation } \\
\hline Only Hispanic & 19 & 7 \\
\hline More Hispanic than Anglo & 220 & 84 \\
\hline Both Hispanic and Anglo & 11 & 4 \\
\hline More Anglo than Hispanic & 13 & 5 \\
\hline \multicolumn{3}{|l|}{ Language preference } \\
\hline Spanish & 175 & 64 \\
\hline English & 8 & 3 \\
\hline Both & 91 & 33 \\
\hline \multicolumn{3}{|l|}{ LPQ } \\
\hline \multicolumn{3}{|l|}{ Birth place } \\
\hline Puerto Rico & 243 & 92 \\
\hline Other & 20 & 8 \\
\hline \multicolumn{3}{|l|}{ Travel to USA } \\
\hline Yes & 229 & 83 \\
\hline No & 40 & 15 \\
\hline \multicolumn{3}{|l|}{ Family in USA } \\
\hline Yes & 228 & 83 \\
\hline No & 40 & 15 \\
\hline \multicolumn{3}{|l|}{ Desire to live in USA } \\
\hline Yes & 113 & 41 \\
\hline No & 160 & 59 \\
\hline \multicolumn{3}{|l|}{ Foresee settling in USA } \\
\hline Yes & 90 & 35 \\
\hline No & 166 & 65 \\
\hline
\end{tabular}

Note. PAS: Psychological Acculturation Scale; LPQ: Lifestyle Preference Questionnaire.

island and self-identified as Puerto Ricans $(n=12)$ remained in the final sample. Participants ranged from 18 to 77 years of age $(M=35$, S.D. $=15.4)$, and consisted of 153 females and 119 males. Participants were recruited from community centers, churches, and university campuses in order to obtain a more accurate appraisal of distinct cultural characteristics.

As indicated in Table 1, the vast majority of participants reported Puerto Rico as their birth place (92\%). An assessment of language preference within the sample revealed that $64 \%$ preferred Spanish only, 3\% preferred English only, and $33 \%$ preferred both Spanish and English. Eighty-four percent $(n=220)$ of participants reported identifying more with Hispanic/Latino culture than Anglo (American) culture. No participants in this sample reported identifying with Anglo (American) culture only. These findings indicate a high degree of enculturation in this sample. 


\subsection{Measures}

Participants provided relevant demographic information (i.e., age, socioeconomic status) as a means of assessing generalizability of the findings. Comparable Spanish and English versions of the questionnaire were available. None of the participants chose to complete the English version of the questionnaires.

\subsubsection{Anxiety Sensitivity Index (ASI)}

The ASI (Reiss, Peterson, Gursky, \& McNally, 1986) is a 16-item questionnaire in which participants indicate on a 5-point Likert-type scale $(0=$ very little to $4=$ very much) the degree to which they fear anxiety symptoms. Among clinical and non-clinical Caucasian samples, the ASI has been demonstrated to have good internal consistency (range of $\alpha$ coefficients: .79-.90) and good testretest reliability ( $r=.75$ ) Reiss et al., 1986. Past investigations have failed to reach a consensus on the issue of normative ASI data (Peterson \& Plehn, 1999). Nonetheless, findings have shown that in the general population, means vary from 14.2 to 22.5 , with an overall mean of approximately 19. Among Spanish samples, Sandin, Chorot, and McNally (2001) have reported an ASI mean range of $18.8-22.1$.

\subsubsection{State-Trait Anxiety Inventory (STAI)}

The State version (STAI-S) of the STAI is a 20-item questionnaire aimed at assessing current anxiety levels. STAI-S items are rated from 1 (not at all) to 4 (very much so) indicating the level of distress aroused by each statement. The Trait version (STAI-T) of the STAI is a 20-item questionnaire designed to assess a respondent's general level of anxiety. General affective tendencies are determined by how an individual rates each item on a scale from 1 (almost never) to 4 (almost always). Equivalent to the English versions, both sets of Spanish measures have been shown to possess high internal consistency (range of $\alpha$ coefficients: .82-.95) (Knight, Waal-Manning, \& Spears, 1983; Spielberger, Gorsuch, \& Lushene, 1970; Spielberger, 1971; Virella, Arbona, \& Novy, 1994). Among a non-clinical sample of university Puerto Rican students, mean scores have been reported to be 36 (S.D. = 10) on the STAI-S and 38 (S.D. = 9.1) on the STAI-T (Virella et al., 1994).

\subsubsection{Beck Anxiety Inventory (BAI)}

The BAI is a 21-item questionnaire aimed at measuring anxiety related symptoms experienced by an individual over the past week. Respondents rate each item using a Likert-type scale ranging from 0 (none) to 3 (always). A mean score of 6, with a standard deviation of 8, has been reported for a mostly European American non-disordered population (Gillis, Haaga, \& Ford, 1995), in addition to an internal consistency coefficient of .92 (Beck, Epstein, Brown, \& Steer, 1988). Among a non-clinical sample of Hispanics, investigations have found a mean score of approximately 23 (S.D. = 14.8) (Novy, Stanley, Averill, \& Daza, 2001). 


\subsubsection{Beck Depression Inventory (BDI-II)}

The revised version of the BDI (Beck, Steer, \& Brown, 1996) is a 21-item measure designed to assess intensity of depressive symptoms during the past week. Scores range from 0 to 63 , with guidelines categorizing respondents within a five level range of severity of depression. A meta-analysis of the BDI's reliability estimates reported a mean $\alpha$ coefficient of .81 for non-psychiatric samples (Beck, Steer, \& Garbin, 1988). Inclusion of the BDI-II allowed for assessment of the discriminant validity of the ASI.

An investigation on the psychometric properties of the Spanish BDI-II among a non-clinical Hispanic sample found a mean score of 25 (S.D. = 13.6; Novy et al., 2001).

\subsubsection{Psychological Acculturation Scale (PAS)}

The PAS is an acculturation assessment tool with items pertaining to the individual's sense of psychological attachment or belonging within AngloAmerican and Hispanic culture, as well as an individuals' psychological negotiation between these two cultural entities (Tropp, Erkut, Garcia Coll, Alarcón, \& Vazquez Garcia, 1999). The PAS was developed to emphasize psychological aspects of acculturation rather than the typical behavioral and attitudinal manifestations associated with acculturation scales. The PAS encompasses four dimensions shown to reflect psychological responses to cultural exposure. Cultural Loyalty assesses an individual's sense of commitment toward the preferred culture. A sample item from this dimension is "Which culture do you feel proud to be part of?" Solidarity assesses an individual's sense of cultural cohesiveness, as reflected by the following sample item "With which group of people do you feel you share most of your beliefs and values?" Comprehension assesses an individual's belief of the understanding achieved regarding values, morals, attitudes, and behaviors culturally sanctioned by the group with which there has been identification. A sample item representative of this dimension is "In which culture do you know what is expected of a person in various situations?" Finally, Identification assesses the manner in which an individual equates himself or herself to the culture of choice. A sample item from this dimension is "In your opinion, which group of people best understands your ideas, your ways of thinking?"

High levels of internal consistency and construct validity were established among a sample of bilingual adolescents and adults of Puerto Rican descent (Tropp et al., 1999). The PAS consists of 12-items intended to reflect an individual's psychological responses to differing cultural contexts. Items are rated on a 5-point Likert-type scale ranging from 1 (only Hispanic/Latino) to 5 (only Anglo/American), with a bicultural orientation at the midpoint. As such, a bicultural orientation (both equally/mixed Hispanic/Latino and Anglo/American) may be characterized as a comparable sense of connection between both cultures (see Table 1). 


\subsubsection{Lifestyle Preference Questionnaire ( $L P Q)$}

The LPQ, a measure developed for this study, was designed to further assess an individual's cultural preference. The LPQ assesses an individual's birthplace (Puerto Rico, other), previous travel to the United States, whether they have family residing in the United States, desire to reside in the United States, and whether they foresee settling in the United States. Each item is responded to as either "yes" or "no." The purpose of this measure is to gain a better understanding of an individual's sense of ethnic identity by gathering information on pragmatic lifestyle choices.

\subsection{Procedure}

Participants who voluntarily agreed to take part in the study by granting consent were asked to complete the battery of self-report measures. Completion of the measures, which were randomized to prevent order effects, lasted approximately $45 \mathrm{~min}$. Upon completion of the questionnaires, subjects were debriefed. As an incentive to volunteer, groups of 50 participants who completed a questionnaire packet were entered into a drawing for a US $\$ 50$ prize.

\section{Results}

\subsection{Descriptive statistics}

Descriptive information is presented in Table 2. In general, this sample was predominantly female $(55.6 \%)$, with an average age of 35 years $($ S.D. $=15.4)$. Scores on the ASI approximate a normal distribution with the majority of scores (75\%) falling between 15 and 35, and approximately $8 \%$ falling below and above a score of 10 and 40, respectively. Mean ASI scores were higher than those typically reported for mostly European American samples $(M=19.01$, S.D. $=9.11$; Peterson \& Plehn, 1999) and non-clinical Spanish samples $(M=20.0$, S.D. $=9.0$; Sandin et al., 2001). Similarly, BAI scores were elevated and comparable to scores for clinical samples (e.g., mean for patients with panic disorder are approximately 27). Means scores on STAI-S and STAI-T were lower than norms for mostly European American populations, but comparable to norms reported for Hispanic samples (STAI-S, $M=36$; STAI-T, $M=38$ ) (Virella et al., 1994). BDI mean scores are comparable to those of non-depressed predominantly European American populations, and lower than scores found among nondepressed Hispanics $(M=25.0$, S.D. $=13.6$; Novy et al., 2001).

\subsection{Confirmatory factor analyses}

As this was the first study to examine the factor structure of the ASI among island Puerto Ricans, a confirmatory factor analysis (CFA) was computed to 
Table 2

Descriptive statistics

\begin{tabular}{|c|c|c|}
\hline \multirow[t]{2}{*}{ Measure } & \multicolumn{2}{|l|}{ Data } \\
\hline & $M$ & S.D. \\
\hline \multirow[t]{2}{*}{ Age (years) } & 35.07 & 15.4 \\
\hline & $n$ & $\%$ \\
\hline \multicolumn{3}{|l|}{ Gender } \\
\hline Female & 153 & 55.6 \\
\hline Male & 119 & 43.3 \\
\hline \multicolumn{3}{|c|}{ Annual family income } \\
\hline$>10,000$ & 33 & 12.8 \\
\hline $10,000-19,000$ & 51 & 19.8 \\
\hline $20,000-29,000$ & 49 & 19.0 \\
\hline $30,000-39,000$ & 30 & 11.6 \\
\hline $40,000-49,000$ & 21 & 8.1 \\
\hline $50,000-59,000$ & 10 & 3.9 \\
\hline $60,000-80,000$ & 24 & 9.3 \\
\hline \multirow[t]{2}{*}{$>80,000$} & 40 & 15.5 \\
\hline & $M$ & S.D. \\
\hline ASI & 24.6 & 13.9 \\
\hline STAI-T & 33.7 & 9.24 \\
\hline STAI-S & 35.1 & 10.50 \\
\hline BAI & 30.6 & 9.65 \\
\hline BDI-II & 7.16 & 7.22 \\
\hline PAS & 25 & 7.81 \\
\hline
\end{tabular}

Note. ASI: Anxiety Sensitivity Index; STAI-S: State-Trait Anxiety Inventory-State version; STAIT: State-Trait Anxiety Inventory-Trait version; BAI: Beck Anxiety Inventory; BDI-II: Beck Depression Inventory—Second Edition; PAS: Psychological Acculturation Scale.

assess the goodness-of-fit of this data to that referenced in the literature as typical of predominantly European American and Spanish samples (Sandin et al., 1996; Zinbarg et al., 1997).

The data were initially fitted to the three factor pattern matrix reported by Zinbarg et al. (1997), delineated by three distinguishable dimensions: Physical Concerns (ASI Items 3, 4, 6, 8, 9, 10, 11, 14), Mental Incapacitation (ASI Items $2,12,15,16$ ), and Social Concerns (ASI Items 1, 5, 13). This threefactor model proved to be a poor fit as indicated by the various fit indices employed (i.e., Chi-square, root mean square residual (RMSR), goodness-of-fit index (GFI), and the adjusted goodness-of-fit index (AGFI)). Crowley and Fan (1997) noted that acceptable GFI and AGFI values are typically higher than the .90 cutoff. Chi-square results indicated a significant distinction between Zinbarg et al.'s factorial model (1997) and the present data (see Table 3). A second CFA conducted to assess the fit of our data to Sandin et al.'s (1996) Spanish single factor structure also indicated a poor fit with the current data. 
Table 3

Goodness-of-fit from confirmatory factor analysis

\begin{tabular}{llrrrrr}
\hline Model & $\chi^{2}$ & \multicolumn{1}{c}{$d f$} & $P$ & RMR & GFI & AGFI \\
\hline Zinbarg, Barlow, and Brown (1997) & 518.67 & 88 & .00 & .276 & .803 & .732 \\
Sandin et al. (1996) & 544.03 & 104 & .00 & .114 & .781 & .714 \\
\hline
\end{tabular}

Note. RMR: root mean square residual; GFI: goodness-of-fit index; AGFI: adjusted goodness-of-fit index.

\subsection{Exploratory factor analysis}

As CFA results suggested that previous single-and three-factor solution models did not provide a good fit for the present data, an exploratory factor analysis (EFA) with oblique rotation was conducted. An examination of the scree plot and eigenvalues greater than 1 (in descending order: $7.90,1.29,1.16, .83, .71, .60, .52$, $.48, .45, .39, .37, .33, .31, .27, .21$, and .18 ), initially suggested the presence of three factors. However, given that all items on Factor 1 also loaded on Factor 3, and vice versa, we chose to interpret the items that shared loadings as a single factor, resulting in a two-factor solution. Further support for the two-factor interpretation of the data is provided by the percentage of variance captured in a two-factor solution which remained substantial (58\%) and the relatively high internal consistency of each of the two factors (see Table 4).

Loadings of the variables on each factor are shown in Table 5. Subsuming biloading items onto one factor allowed us to interpret the first factor as Anxiety Sensitivity. This Anxiety Sensitivity Factor encompassed 14 of the 16 ASI items. It is noteworthy to highlight Items 3, 12, 2, and 13. Items 3, 12 and 13 loaded onto both extracted factors. However, because there was a stronger loading onto Factor 1 , it was determined that these should be maintained as part of this factor. Item 2 was the only item to load solely onto Factor 3 . Internal consistency remained high (.92) even when this item was removed form Factor 1, as such it was determined that this item remained as part of Factor 1.

Factor 2 (Items 1 and 5) in the present data set overlaps with Zinbarg et al.'s (1997) Factor 3 Social Concerns (ASI Items 1, 5, 13). Notably, Item 13 ("Other people notice when I feel shaky") was also associated with Factor 1, Anxiety Sensitivity.

Table 4

Variance explained by a two-factor exploratory factor analysis

Initial eigenvalues

Alpha internal

\begin{tabular}{lllll}
\hline Factor & Total & \% of variance & Cumulative $(\%)$ & consistency \\
\hline 1 & 7.939 & 49.62 & 49.62 & .93 \\
2 & 1.297 & 8.11 & 57.73 & .72 \\
\hline
\end{tabular}


Table 5

ASI factor loadings

\begin{tabular}{|c|c|c|c|c|}
\hline \multirow[t]{2}{*}{ Item } & & \multicolumn{3}{|c|}{ Factor } \\
\hline & & 1 & 2 & 3 \\
\hline 6 & It scares me when my heart beats rapidly & .871 & .319 & -.44 \\
\hline 10 & It scares me when I become short of breath & .839 & .344 & -.447 \\
\hline 9 & $\begin{array}{l}\text { When I notice that my heart is beating rapidly, } \\
\text { I worry that I might have a heart attack }\end{array}$ & .844 & .225 & -.433 \\
\hline 11 & When my stomach is upset, I worry that I might be seriously ill & .787 & .273 & -.545 \\
\hline 8 & It scares me when I am nauseous & .734 & .307 & -.542 \\
\hline 7 & It embarrasses me when my stomach growls & .695 & .318 & -.564 \\
\hline 4 & It scares me when I feel faint & .67 & .36 & -.421 \\
\hline 14 & Unusual body sensation scare me & .699 & .319 & -.682 \\
\hline 1 & It is important to me not to appear nervous & .303 & .873 & -.313 \\
\hline 5 & It is important to me to stay in control of my emotions & .432 & .848 & -.327 \\
\hline 16 & It scares me when I am nervous & .696 & .317 & -.819 \\
\hline 3 & It scares me when I feel "shaky" (trembling) & .529 & .494 & -.784 \\
\hline 15 & When I am nervous, I worry that I might be mentally ill & .534 & & -.775 \\
\hline 12 & It scares me when I am unable to keep my mind on a task & .581 & .404 & -.736 \\
\hline 2 & $\begin{array}{l}\text { When I cannot keep my mind on a task, I worry that } \\
\text { I might be going crazy }\end{array}$ & .329 & .237 & -.746 \\
\hline 13 & Other people notice when I feel shaky & .534 & .483 & -.706 \\
\hline 14 & Unusual body sensation scare me & .699 & .319 & -.682 \\
\hline
\end{tabular}

Note. Factor values $>.40$ are shown in boldface.

\subsection{Convergent and discriminant validity}

To examine the relationship between the ASI and the convergent and discriminant validity measures, Pearson's correlations were calculated (see Table 6).

Table 6

Pearson's correlations among dependent variables

\begin{tabular}{|c|c|c|c|c|c|c|c|c|}
\hline Variable & ASI & Factor 1 & Factor 2 & BAI & STAI-S & STAI-T & BDI-II & PAS \\
\hline ASI & - & & & & & & & \\
\hline Factor 1 & $.99^{* *}$ & - & & & & & & \\
\hline Factor 2 & $.62^{* *}$ & $.49^{* *}$ & - & & & & & \\
\hline BAI & $.55^{* *}$ & $.56^{* *}$ & $.31^{* *}$ & - & & & & \\
\hline STAI-S & $.31^{* *}$ & $.33^{* *}$ & .09 & $.47^{* *}$ & - & & & \\
\hline STAI-T & $.44^{* *}$ & $.45^{* *}$ & $.17^{*}$ & $.47^{* *}$ & $.69^{* * *}$ & - & & \\
\hline BDI-II & $.32^{* *}$ & $.31^{* *}$ & $.16^{*}$ & $.45^{* *}$ & $.55^{* * *}$ & $.61^{* * *}$ & - & \\
\hline PAS & $-.15^{*}$ & $-.17^{* *}$ & -.01 & $-.15^{*}$ & $-.19^{* *}$ & $-.18^{*}$ & -.04 & - \\
\hline
\end{tabular}

Note. ASI: Anxiety Sensitivity Index; Factor $1=$ Anxiety Sensitivity; Factor $2=$ Emotional Concerns; BAI: Beck Anxiety Inventory; STAI-S: State-Trait Anxiety Inventory-State version; STAI-T: State-Trait Anxiety Inventory_-Trait version; BDI-II: Beck Depression Inventory-Second Edition; PAS: Psychological Acculturation Scale.

$$
\begin{aligned}
& { }^{*} P<.05 . \\
& { }^{* *} P<.01 .
\end{aligned}
$$


Results indicated that the ASI was significantly correlated with all measures of anxiety and depression, as was Factor $1(P>.01)$. The correlations of both scales with trait measures of anxiety (i.e., BAI, STAI-T) were significantly higher than the correlation between each scale and the BDI providing some evidence of discriminant validity. Alternatively, Factor 2 was not correlated to the STAI-S and the BDI, while being correlated to the BAI and STAI-T $(r=.31$ and .17 , respectively). While suggestive of discriminant validity, evidence for the convergent validity of Factor 2 is weak. Further analyses revealed a significant negative correlation between acculturation and the anxiety scales (range from -.15 ASI to -.19$)$ but not with depression $(r=-.04)$. This inverse relationship supports the initial hypothesis that as acculturation increases, the experience of overall anxiety-related symptoms is reduced.

\section{Discussion}

Results from this study were inconsistent with previous investigative efforts, which found evidence for single- and three-factor ASI solutions in predominantly European American and Spanish samples, respectively (Sandin et al., 1996; Zinbarg et al., 1997). Results from CFA revealed that neither solution provided a suitable fit for the present data set. An initial EFA extracted a three-factor solution that accounted for a significant percent of the variance. An examination of the composition of each factor, however, indicated that all of the items on Factor 1 also loaded on Factor 3, with the reverse being true. Therefore, the bulk of items were considered as being part of the same underlying factor, Anxiety Sensitivity.

This interpretation is consistent with the work of Sandin et al. (1996), who found a one-factor solution, as well as previous researchers who have discussed the overlap between psychological and somatic sensations among Hispanics. Integration of somatic and psychological forms of expression reflects the sociocultural context characteristic of Hispanic populations and also provides further evidence for the need to reevaluate diagnostic categories. Thus, subsuming these 14 items onto a single factor allows one to explore the plausibility of cultural conceptualizations rooted in the idea that mental illness among Hispanic populations is largely recognized as a somatic construct (Sylva, 1997). For example, Item 16 ("It scares me when I am nervous"), typically associated with Mental Incapacitation concerns among European Americans, may point to a combined psychobiological interpretation by Puerto Ricans.

One exception that should be noted is the initial loading of Item 2 ("When I cannot keep my mind on a task, I worry that I might be going crazy") onto Factor 3. It is possible that this item is a poor fit with anxiety sensitivity among Puerto Ricans. It is further possible that a true third factor exists but that the limited content of the 16-item ASI does not allow this factor to emerge. When Item 2 was removed from Factor 1, the internal consistency remained high (.92), suggesting 
that this item may not be a significant part of the factor, or anxiety sensitivity among Puerto Rican populations.

The second interpretable factor consisted of Items 1 and 5. It is likely that these items reflect an issue of emotional control similar to the findings of Carter and colleagues (1999) with respect to African Americans. The significance of staying emotionally in control may be a reflection of a Puerto Rican's fear of being stigmatized as mentally ill. Research suggests that a significant number of Hispanics believe that being labeled as mentally ill implies that they "are crazy" (estar loco) or somehow genetically defective (LaBruzza \& Méndez-Villarrubia, 1997). Thus, the argument can be made that for Puerto Ricans being emotionally in control stems from the deeply ingrained stereotype that going "loco" is shameful and a source of inferiority. It is possible that the concern may reduce their self-reported experience of anxiety. In this case the prevalence rates of anxiety disorders may in actuality be higher than current estimates. This interpretation, however, is made with some caution since this factor only contains two items.

As expected, significant correlations between the ASI and the BAI, STAI-S and STAI-T provided strong evidence for convergent validity. The significant correlation between the ASI and the BDI-II suggests poor discriminant validity. It should be noted, however, that the magnitude of these correlations were smaller than those found between the ASI and trait anxiety scales. Furthermore, previous investigations have found that the STAI-T contains elements of both anxiety and depression (Bieling, Antony, \& Swinson, 1998; Schmidt, Lerew, \& Joiner, 1998; Taylor, Koch, Woody, \& McLean, 1996). Thus, it may be possible that the measures employed cannot accurately differentiate between anxiety and depression. It is also plausible that the relationship between anxiety and depression among Puerto Ricans may not be as distinctly expressed as it is among European Americans.

Consistent with the initial hypotheses, acculturation level was significantly negatively correlated with overall anxiety measures. Specifically, it was shown that higher levels of acculturation to Anglo/American culture are associated with lower AS. Thus, the more strongly an individual identified himself with Hispanic values, beliefs, and ideals, the greater the reported fear of anxiety symptoms. This inverse relationship held on all dependent measures of anxiety, yet was not observed on a measure of depression. This suggests that culture may play a significant role in the expression of anxious pathology, while bearing no significance on depressive symptomatology. This finding is consistent with research indicating higher levels of anxiety disorders among Puerto Ricans (Canino et al., 1987). Our findings further support this notion as mean BAI and ASI scores among our sample were higher than normative sample data. It could also be speculated that Puerto Ricans display a greater willingness to report anxious phenomena, more specifically nervousness, than depressive symptomatology. This would be consistent with certain cultural tenets which center around the belief that it is more socially acceptable to express nervousness than the depressive symptoms, the latter of which are considered to be indicative of 
weakness. These findings are contrary to Mirowsky and Ross (1987) who reported that Mexicans raised in Mexico showed lower levels of anxiety and depression than those raised in the United States. A possible explanation for this variation may lie in the fact that within Hispanic groups there might exist distinct cultural patterns of distress.

It is imperative to examine alternative explanations that could be responsible for the inconsistency of factor structures found between the present study and those reported by Zinbarg et al. (1997) and Sandin et al. (1996). ASI score variability, age composition of samples, and gender effects across these three studies could all contribute to the differential structures discovered. However, upon close examination, it appears that all three samples, within limits, are roughly equivalent. In terms of age distribution, the present sample is comparable to Sandin et al.'s (1996) Spanish sample whose mean age is approximately 30. Likewise, ASI score variability appears to be irrelevant. Finally, there appear to be some significant gender differences in the current sample, however the lack of information available on gender differences, if any, on Sandin et al.'s (1996) sample does not allow us to further investigate this possibility.

It is also important to note some of the limitations of exploratory factor analysis. First, it is worth noting that EFA capitalizes in chance variations of the original correlation matrix, and therefore, can be problematic, particularly in terms of making generalizations to other samples. Second, using this approach, Pearson product-moment correlation coefficients are computed from responses made on a Likert-type scale. The normality of distribution and homogeneity of variance cannot necessarily be assumed for Likert-type scales. Aware of this caveat we computed a homogeneity of variance test and results suggest that our sample does show normal distribution.

Results from this study, however, do not allow strong conclusions as to whether the pattern of results is influenced by cultural identity or level of acculturation. Future efforts should examine the factor structure of the ASI among Hispanic samples with diverse acculturative levels. This would allow one to address whether the latent factor structure of the ASI among this sub-sample will more closely resemble that found for predominantly European American samples, or if ASI score variability differs as a function of acculturation.

Future studies should also focus on examining the factor structure of the ASI among Puerto Rican clinical samples. Although measures of anxious pathology were gathered, this study did not assess for history of panic or anxious pathology, making it difficult to generalize our findings to clinical samples. Similarly, forthcoming investigations ought to consider the role a history of psychopathology or ataques de nervios might play in the distribution of ASI scores among a non-clinical sample. Similarly, studies should focus on how factor analytic structures would materialize across an array of psychopathological phenotypes among this population.

Furthermore, potential research efforts should note the possible presence of self-selection biases in the current sample, in addition to establishing greater 
controls for SES and education level. The sample in this investigation was somewhat more educated and economically affluent than the census data shows for island Puerto Ricans. Previous research has shown that depressive symptoms are less related to a diagnosis of depression among economically disadvantaged Hispanics than for those who are more socially advantaged (Cho et al., 1993). Lastly, findings of this study may only be potentially applicable to those Puerto Ricans residing in the metropolitan area of the island. The geographical focus should be expanded in order to get a more accurate picture generalizable to the entire island.

Future studies will need to confirm, by replication, the ASI two-factor solution by way of CFA. Similarly, our results point to the need to employ the extended version of the ASI to assess whether the items in this measure add additional support for a multifactor solution among Hispanic populations. It is possible that the extended ASI version taps into constructs that the 16-item version fails to encompass. It would be noteworthy to examine gender differences in factor composition of the ASI, in addition to examining a variety of cultural groups within the Hispanic umbrella. Finally, there is virtually no research on the concept of ataques de nervios as it relates to anxiety sensitivity. Thus, if theories to date are indeed accurate, factor structures of the ASI among Hispanics will be similar, but in some ways distinct different from that of European American and African Americans.

\section{References}

Aponte, J. F., \& Wohl, J. (Eds.). (2000). Psychological intervention and cultural diversity. Massachusetts: Allyn \& Bacon.

Beck, A. T., Epstein, N., Brown, G., \& Steer, R. A. (1988). An inventory for measuring clinical anxiety: psychometric properties. Journal of Consulting and Clinical Psychology, 56, 893897.

Beck, A. T., Steer, R. A., \& Brown, G. K. (1996). Beck Depression Inventory-Second Edition (BDIII) manual. San Antonio: The Psychological Corporation.

Beck, A. T., Steer, R. A., \& Garbin, M. G. (1988). Psychometric properties of the Beck Depression Inventory: twenty-five years of evaluation. Clinical Psychology Review, 8, 77-100.

Bieling, P. J., Antony, M. M., \& Swinson, R. P. (1998). The State-Trait Anxiety Inventory, Trait version: structure and content re-examined. Behaviour Research and Therapy, 36, 777-788.

Canino, G., Bird, H. R., Shrout, P. E., Rubio-Stipec, M., Navarro, M., Martínez, R. et al. (1987). The prevalence of specific psychiatric disorders in Puerto Rico. Archives of General Psychiatry, 44, 727-735.

Carter, M. M., Miller, O., Jr., Sbrocco, T., Suchday, S., \& Lewis, E. L. (1999). Factor structure of the Anxiety Sensitivity Index among African American college students. Psychological Assessment, $11,525-533$.

Carter, M. M., Sbrocco, T., \& Carter, C. (1996). African Americans and anxiety disorders research: development of a testable theoretical framework. Psychotherapy: Theory, Research, \& Practice, 33, 449-463.

Cho, M. J., Moscicki, E. K., Narrow, W. E., Rae, D. S., Locke, B. Z., \& Regier, D. A. (1993). Concordance between two measures of depression in the Hispanic health and nutrition examination survey. Social Psychiatry and Psychiatric Epidemiology, 28, 156-163. 
Crowley, S. L., \& Fan, X. (1997). Structural equation modeling: basic concepts and applications in personality assessment research. Journal of Personality Assessment, 68, 508-531.

Dohrenwend, B. P. (1969). Social status and psychological disorder: an issue of substance, an issue of method. American Sociology Review, 31, 14-34.

Donnell, C. D., \& McNally, R. J. (1990). Anxiety sensitivity and panic attacks in a nonclinical population. Behaviour Research and Therapy, 28, 83-85.

Duran, D. G. (1995). The impact of depression, psychological factors, cultural determinants, and the patient/care-provider relationship on somatic complaints of the distressed Latina. Dissertation Abstracts International, 56(6-A), 2428.

Escobar, J. E., Randolph, E. T., Puente, G., Spiwak, F., Asamen, J. K., Hill, M. et al. (1983). Posttraumatic stress disorder in Hispanic Vietnam veterans: clinical phenomenology and sociocultural characteristics. Journal of Nervous and Mental Health, 171, 585-596.

Flaherty, J. A. (1987). Appropriate and inappropriate research methodologies for Hispanic mental health. In: M. Gaviria \& J. D. Arana (Eds.), Health and behavior: research agenda for Hispanics (pp. 177-186). The University of Illinois at Urbana-Champaign: Publication Services.

Gaw, A. C. (Ed.). (1993). Culture, ethnicity, and mental illness. Washington, DC: American Psychiatric Press, Inc.

Gillis, M. M., Haaga, D. A., \& Ford, G. T. (1995). Normative values for the Beck Anxiety Inventory, fear questionnaire, Penn State worry questionnaire, and social phobia and anxiety inventory. Psychological Assessment, 7, 450-455.

Haberman, P. W. (1976). Psychiatric symptoms among Puerto Ricans in Puerto Rico and New York city. Ethnicity, 3, 133-144.

Karno, M., Burnam, M. A., Hough, R. L., Escobar, J. I., \& Golding, J. M. (1987) Mental disorders among Mexican Americans and non-Hispanic Whites in Los Angeles. In: M. Gaviria \& J. D. Arana (Eds.), Health and behavior: research agenda for Hispanics (pp. 110-126). The University of Illinois at Urbana-Champaign: Publication Services.

Knight, R. G., Waal-Manning, H. J., \& Spears, G. F. (1983). Some norms and reliability data for the State-Trait Anxiety Inventory and the Zung Self-Rating Depression scale. British Journal of Clinical Psychology, 22, 245-249.

LaBruzza, A. L., \& Méndez-Villarrubia, J. M. (1997). Using DSM-IV: a clinician's guide to psychiatric diagnosis. New Jersey: Jason Aronson Inc.

Maller, R. G., \& Reiss, S. (1992). Anxiety sensitivity in 1984 and panic attacks in 1987. Journal of Anxiety Disorders, 6, 241-247.

Mirowsky, J., \& Ross, C. E. (1987). Support and control in Mexican and Anglo cultures. In: M. Gaviria \& J. D. Arana (Eds.), Health and behavior: research agenda for Hispanics (pp. 85-92). The University of Illinois at Urbana-Champaign: Publication Services.

Mohlman, J., \& Zinbarg, R. (2000). The structure and correlates of anxiety sensitivity in older/adults. Psychological Assessment, 12, 440-446.

Novy, D. M., Stanley, M. A., Averill, P., \& Daza, P. (2001). Psychometric comparability of Englishand Spanish-language measures of anxiety and related affective symptoms. Psychological Assessment, 13, 347-355.

Ortega, A. N., Rosenheck, R., Alegria, M., \& Desai, R. A. (2000). Acculturation and the lifetime risk of psychiatric and substance use disorders among Hispanics. The Journal of Nervous and Mental Disease, 188, 728-735.

Peterson, R. A., \& Plehn, K. (1999). Measuring anxiety sensitivity. In: S. Taylor (Ed.), Anxiety sensitivity: theory, research, and treatment of the fear of anxiety (pp. 61-81). Mahwah, NJ: Lawrence Erlbaum Associates.

Reiss, S. (1991). Expectancy theory of fear, anxiety and panic. Clinical Psychology Review, 11, 141153.

Reiss, S., Peterson, R. A., Gursky, D. M., \& McNally, R. J. (1986). Anxiety sensitivity, anxiety frequency and the prediction of fearfulness. Behavior Research and Therapy, 24, 1-8. 
Rogler, L. H., Cortes, D. E., \& Malgady, R. G. (1991). Acculturation and mental health status among Hispanics: convergence and new directions for research. American Psychologist, 46, 585-597.

Rogler, L. H., Gurak, D. T., \& Santana Cooney, R. (1987). The migration experience and mental health: formulations relevant to Hispanics and other immigrants. In: M. Gaviria \& J. D. Arana (Eds.), Health and behavior: research agenda for Hispanics (pp. 72-84). The University of Illinois at Urbana-Champaign: Publication Services.

Sandin, B., Chorot, P., \& McNally, R. J. (1996). Validation of the Spanish version of the Anxiety Sensitivity Index in a clinical sample. Behavior Research and Therapy, 34, 283-290.

Sandin, B., Chorot, P., \& McNally, R. J. (2001). Anxiety Sensitivity Index: normative data and its differentiation from trait anxiety. Behavior Research and Therapy, 39, 213-219.

Schmidt, N. B., Lerew, D. R., \& Joiner, T. E. (1998). Anxiety sensitivity and the pathogenesis of anxiety and depression: evidence for symptom specificity. Behavior Research and Therapy, 36, $165-177$.

Smith, L. C., Friedman, S., \& Nevid, J. (1999). Clinical and sociocultural differences in African American and European American patients with panic disorder and agoraphobia. Journal of Nervous and Mental Disease, 187, 549-560.

Spielberger, C. D. (1971). Development of the Spanish edition of the State-Trait Anxiety Inventory. Revista Interamericana de Psicologia, 5, 145-158.

Spielberger, C. D., Gorsuch, R. L., \& Lushene, R. E. (1970). Manual for the State-Trait Anxiety Inventory. Palo Alto: Consulting Psychologists Press.

Straussner, S. L. A. (Ed.). (2001). Ethnocultural factors in substance abuse treatment. New York: The Guilford Press.

Sylva, A. (1997). Inter-ethnic and intra-cultural differences on somatic and psychological/affective expressions of emotional distress: a case study of Puerto Rican, non-Hispanic Black and nonHispanic White women in the South Bronx. Dissertation Abstracts International, 58(4-A), 1456.

Taylor, S., Koch, W. J., McNally, R. J., \& Crockett, D. J. (1992). Conceptualizations of anxiety sensitivity. Psychological Assessment, 4, 245-250.

Taylor, S., Koch, W. J., Woody, S., \& McLean, P. (1996). Anxiety sensitivity and depression: how are they related? Journal of Abnormal Psychology, 105, 474-479.

Tropp, L. R., Erkut, S., Garcia Coll, C., Alarcon, O., \& Vazquez Garcia, H. A. (1999). Psychological acculturation: development of a new measure for Puerto Ricans on the U.S. mainland. Educational and Psychological Measurement, 59, 351-367.

U.S. Bureau of the Census. (n.d.). Profile of general demographic characteristics: 2000. Retrieved February 2003, from http://factfinder.census.gov/servlet/BasicFactsServlet.

Virella, B., Arbona, C., \& Novy, D. (1994). Psychometric properties and factor structure of the Spanish version of the State-Trait Anxiety Inventory. Journal of Personality Assessment, 63, 401-412.

Zinbarg, R. E., \& Barlow, D. H. (1996). Structure of anxiety and the anxiety disorders: a hierarchical model. Journal of Abnormal Psychology, 105, 181-193.

Zinbarg, R. E., Barlow, D. H., \& Brown, T. A. (1997). Hierarchical structure and general factor saturation of the Anxiety Sensitivity Index: evidence and implications. Psychological Assessment, 9, 277-284.

Zinbarg, R. E., Mohlman, J., \& Hong, N. N. (1999). Dimensions of anxiety sensitivity. In: S. Taylor (Ed.), Anxiety sensitivity: theory, research, and treatment of the fear of anxiety (pp. 83-114). Mahwah, NJ: Lawrence Erlbaum Associates.

Zvolensky, M. J., Arrindell, W. A., Taylor, S., Bouvard, M., Cox, B. J., Stewart, S. H., Sandin, B., Cardenas, S. J., \& Eifert, G. H. (2003). Anxiety sensitivity in six countries. Behavior Research and Therapy, 41, 841-859. 